\title{
Dynamics of Synaptic Transfer in Living and Simulated Neurons ${ }^{\ddagger}$
}

\author{
Michael Stiber ${ }^{*}$ and José P. Segundo ${ }^{\dagger}$
}

Technical Report HKUST-CS92-5

October 1992

\author{
${ }^{*}$ Department of Computer Science \\ The Hong Kong University of Science and Technology \\ Clear Water Bay, Kowloon, Hong Kong \\ ${ }^{\dagger}$ Department of Anatomy and Cell Biology \\ and Brain Research Institute \\ University of California \\ Los Angeles, California 90024, USA
}

\footnotetext{
${ }^{\ddagger}$ This work was supported by Trent H. Wells, Jr., Inc., the OfßÆeef the Dean of the UCLA School of Medicine (BRSG Funds), the UCLA Department of Anatomy and Cell Biology, and by a grant of computer time made possible under a joint study with the IBM Corporation on the IBM 3090/600J Supercomputer at the UCLA Of/Ece of Academic Computing. Some analysis was performed using NCSA Image and NCSA Layout, developed at the National Center for Supercomputing Applications at the University of Illinois at Urbana-Champaign.
} 


\begin{abstract}
Nervous systems and their constituent neurons often display complex dynamics in response to inputs with simple characteristics. Until recently, these behaviors were not even classiÆedet alone understood. This lack of understanding impedes determination of the utility of dynamical processing elements in artiÆcialeural networks. This paper summarizes a comparison of the responses of an ionic permeability based neural model to periodic inhibitory driving with that of a living preparation. Unlike previous, simpler models, duplication of most neuron response types was excellent, and simulation results led to insights into neuron activities that were subsequently veriÆebly examination of the living data. It is hoped that knowledge of the underlying physiological mechanisms and formal properties of neuron dynamics will lead to advances in artiÆcialeural network computational theory.
\end{abstract}




\section{Introduction}

This paper presents a preliminary comparison of some behaviors found for a dynamic, ionicpermeability-based neural model with those of the crayÆsslowly adapting stretch receptor organ (SAO), both receiving periodic, pacemaker inhibitory input trains. The living preparation is of interest as a prototypical inhibitory synapse, an elementary functional unit in nervous systems. The model allows for exploration of the contributions of underlying mechanisms towards overall neural activity. Nonlinear dynamical analysis techniques used throughout are intended to lead to higher-level descriptions of neural dynamics which may be useful for improving understanding of neural computation.

This work has already illuminated some previously misunderstood neural behaviors. For brevity's sake, details of the model, the living preparation, and data capture and analysis methods, are omitted - they can be found in [?, ?]. It seems that this model is capable of reproducing all behavioral forms found in the SAO, unlike simpler ones based on phase transition curves or leaky integrators [?]. These corresponding model forms contain behavioral details that were either obscured by or confused with noise in the SAO. This includes the presence of chaotic dynamics, which reinforces the idea that the SAO is capable of producing chaotic outputs [?].

\section{Complex Dynamics in Living Neurons}

A neuron produces a series (or train) of short lived pulses, called Action Potentials (APs) as its most obvious output. The timing of the APs, i.e., the times when they arise, are important. Here, we assimilate the train of APs to a point process, with the resultant description the sequence of times of occurrence, $\left\langle t_{0}, t_{1}, \ldots\right\rangle$. From these, one may extract the ordered series of interspike intervals, $\left\langle T_{1}, T_{2}, \ldots\right\rangle, T_{n}=t_{n}-t_{n-1}$.

By way of comparison, the typical output activity of artiÆcialeural network units may be 
either a real or binary value, considered analogous to a neuron’s time-averaged Ærinfgequency, $1 / \hat{T}$, where $\hat{T}$ is a mean over some range of $T_{i}$, computed for a reasonable time interval. Integration of inputs is performed by a weighted sum (with negative weights signifying inhibition), and the resultant output produced by sending the sum through a squashing function, which is nearly linear through much of its range. As a result, an increase in an inhibitory input causes the neuron's output value to monotonically decrease.

However, the result of periodic inhibition of a live pacemaker neuron at different input rates is much more complex. The output rate versus input rate relation includes paradoxical behavior, with a nonmonotonic response curve containing positive-slope locking regions alternating with regions of negative-sloped, non-locked responses [?]. Thus, increasing inhibitory input can either increase or decrease a neuron's Æringate, and different inputs can result in changing output behavioral type. Here we concentrate on introducing three major behavioral categories which the SAO and permeability model have in common.

Any behavior implies telltale relationships between input and output spike timings. Locked forms exhibit a Æxedepeating sequence of $\phi_{i}$ and $T_{i}$; we will call a behavior TMlocked $p: q$ " if these sequences repeat every $q$ outputs and $p$ inputs, so $\phi_{i}=\phi_{i+q}, T_{i}=T_{i+q}$.

Intermittent is a descriptive term used for behaviors which might initially seem locked much of the time, but are interrupted at irregular times by wild deviations in output. Upon closer examination, they are revealed to be not quite periodic, only apparently so. This includes quasiperiodic behaviors, such as phase slidings and walk-throughs, in which $T / I \approx p / q$, for $T / I$ irrational and (relatively small) integer $p, q$. The result is a phase drift or sliding in which phases ${ }^{\mathrm{TM}}$ Walk through" the full range in a non-standard manner.

The third behavior which will be considered has been called TMmessy" because it can't be summarized brieØyr predicted reliably. It includes both erratic and stammering [?]. The erratic case is now considered chaotic [?]. Stammering occurs for high IPSP input frequencies $I<N$, 
in response to which the SAO is only able to produce outputs during narrow ${ }^{\mathrm{TM}}$ windows" of time. Whether the SAO does produce an output during a particular window seems to be random, and has been mostly attributed to the inØuencof noise.

\section{Modeling and Analysis}

The model chosen to match the crayÆs $\mathrm{AAO}$ responses was developed by Edman, Gestrelius, Grampp, and Sjölin for the lobster SAO and FAO (fast adapting stretch receptor organ) [?]. This model, in additional to ion Øoxduring APs, emphasizes between-spike ionic Øuxesnd therefore also includes slow state variables and ionic concentration dependencies. It approximates well the low-frequency spontaneous pacemaker Æringeen in the SAO.

We deal here with analysis of data from forced self-oscillators, with the system under study an intrinsic oscillator, producing periodic output APs. When subjected to periodic forcing inputs, the timing of its output changes, and it exhibits new behaviors. These may differ quantitatively from the unperturbed (regularly spaced APs with different between-AP intervals) or it may differ qualitatively (APs no longer separated by identical intervals). Analyzing these changes is a major focus of this work.

As deÆneih Figure ??, the times of the postsynaptic events are $\left\langle t_{0}, t_{1}, \ldots, t_{n}\right\rangle$, with the interval between two such events $T_{i}=t_{i}-t_{i-1}$. The presynaptic events are of Æxeinterval, $I$, and are used as reference times for analyses. The relation between the two trains is captured by the cross interval between a postsynaptic event and the most recent presynaptic event, $\phi_{i}$, also called the phase, and usually normalized as a fraction of $I$.

A simple (but powerful) way of looking at the relationships between the neuron's current state and a past one is to plot them against each other. In many cases, it is not necessary to use the entire state - one element from it (or some other measured quantity, such as the $T_{i}$ or $\phi_{i}$ ) 


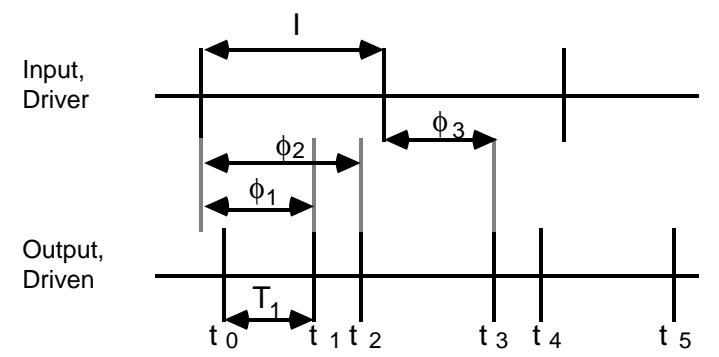

Figure 1: A schematic view of input (presynaptic) and output (postsynaptic) trains. The input events are regular, with interval $I$. The output events may be regular of irregular, with the time of event $i$ designated $t_{i}$ and the interval to preceding one $T_{i}$. Phases $\phi_{i}$ are measured as the time between an output and the immediately preceding input, normalized to $I$.

can be used. For instance, by plotting $\phi_{i}$ versus $\phi_{i+q}$, we obtain a $q^{\text {th }}$ order phase return map.

There are several characteristics of the return map which will be of interest in diagnosing system behavior. First of all, if all of the plotted points fall within $c$ small, discrete clusters in a Ærst-ordemap, then locking would immediately be suspected. We would then proceed to generate the return maps of order $n c$, for some reasonable range of integer $n$, to see if the clusters fall on the diagonal. Clustering of points along the diagonal in the return map is key to locking detection. If $p: q$ locking is exhibited, then the $q^{\text {th }}$ order return map will have all points on the diagonal $\left(\phi_{i+q}=\phi_{i}\right)$. Additionally, these $q$ outputs will occur in the same amount of time that $p$ inputs do, so that $q T=p I$.

When locking is not present, the points in a Ærst-ordereturn map will typically not fall into discrete clusters. If they form a 1-dimensional curve, then additional techniques can be used to analyze behavior. Quasiperiodicity, an irrational driver to driven ratio, results in the systematic progression of $T$ or $\phi$ through the entire range of values, with current state information sufÆcient to uniquely determine the future, and the return map will be a 1-1 invertible mapping. If a 1dimensional curve exists, but it is not $1-1$, or there is no $1-\mathrm{D}$ curve, then there are a variety of possibilities, including stochastic, intermittency, and chaos.

Global behavior addresses the question of how behavior changes as we change the input - 


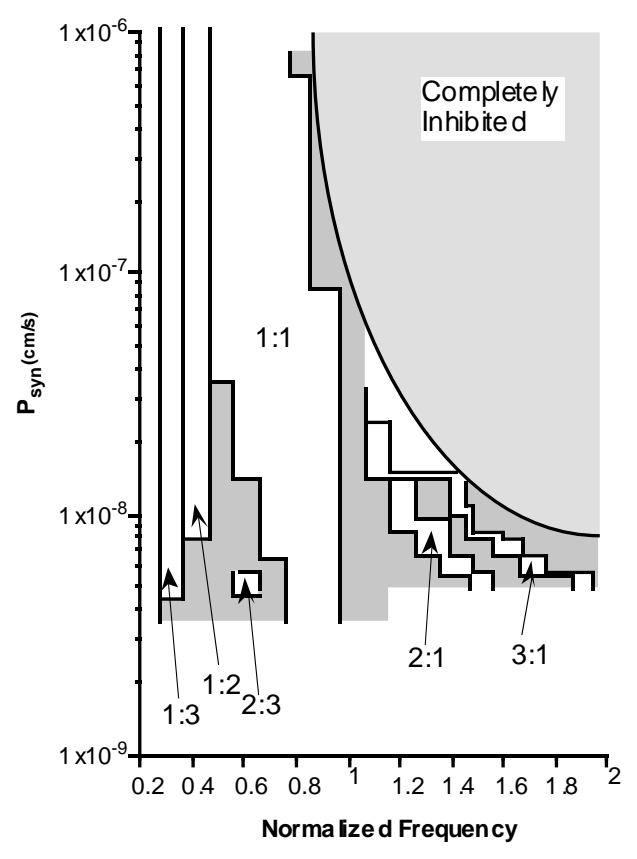

Figure 2: This graph shows the borders of selected locking behaviors for inhibitory input to the permeability model. Cross-hatched areas correspond to other locking ratios or non-locked behavior. Note the region to the right where inhibition shuts the simulated neuron down.

how the different behaviors of the system are related in the space made up of the input (or other) parameters. Here we will be exploring the effects of input amplitude and frequency, and will therefore be constructing two-dimensional Arnol'd maps.

In an Arnol'd map, system behavioral category, such as locked, quasiperiodic, or chaotic, is plotted in the $(F, A)$ plane (where normalized input frequency $F=N / I$, and input amplitude $A=\bar{P}_{s y n}$, measured by the maximum synaptic permeability). Lockings tend to occur in relatively tall, narrow regions in this plane, hence they are given the appellation tongues. We only diagnosed locking to construct approximate maps, and only for a limited number of ratios. Areas between locking tongues may include other lockings or non-locked behaviors. Ranges of input amplitude and normalized frequency were chosen, such that $A_{\min } \leq A \leq A_{\max }$ and $I_{\min } \leq I \leq I_{\max }$. A stepsize was used for both $A$ and $I, A_{\text {step }}$ and $I_{\text {step }}$, so that a simulation was performed at each amplitude step for each frequency step, within the given ranges. 
The map for inhibitory input to the model is Figure ??. Non-locked behaviors occur in the cross-hatched regions, while only the indicated locked forms arose in the white regions. A sparse exploration of the $\left(N / I, \bar{P}_{s y n}\right)$ space was performed, approximately 20 points across and 30 down, for a total of about 500 simulations. It is important to note that such an Arnol'd map cannot be constructed for the SAO itself, for, apart from inevitable time constraints, the preparation's synaptic strength is not an easily controllable variable.

\section{Results}

Several features in the Arnol'd map are immediately apparent. With inhibitory input, the model can be shut down if the input frequency is too high. Additionally, locking tongues tend to narrow at low amplitudes and at high ones (this is noticeable primarily for the 1:1 tongue), the former the result of increasing number of non-locked behaviors, and the latter the ${ }^{\mathrm{TM}}$ squeezing" of the range of frequencies within which the neuron will produce any output at all. With this map as a guide, individual $\left(N / I, \bar{P}_{s y n}\right)$ pairs were explored, and behaviors were found which mimicked well those found in the SAO, i.e. locked (omitted here), intermittent, and messy.

Forcing an oscillator beyond its entrainment limit can result in a type of intermittency called quasiperiodicity, in which an almost (but not quite) locked condition exists [?]. In interval and phase return maps, quasiperiodic behavior results in points on continuous, one-dimensional curves. Phase return maps are also invertible and, since phases ${ }^{\mathrm{TM}}$ walk through" a range of values before (almost) repeating, this behavior is identiÆedith walkthroughs [?], noted also in the SAO.

In the permeability model, two different type of apparently quasiperiodic behavior were found, though they may represent extremes of a range of behaviors. Figure ?? shows example graphs for intervals and phases for the model (A-D) and the SAO (E-H). In both cases, the 

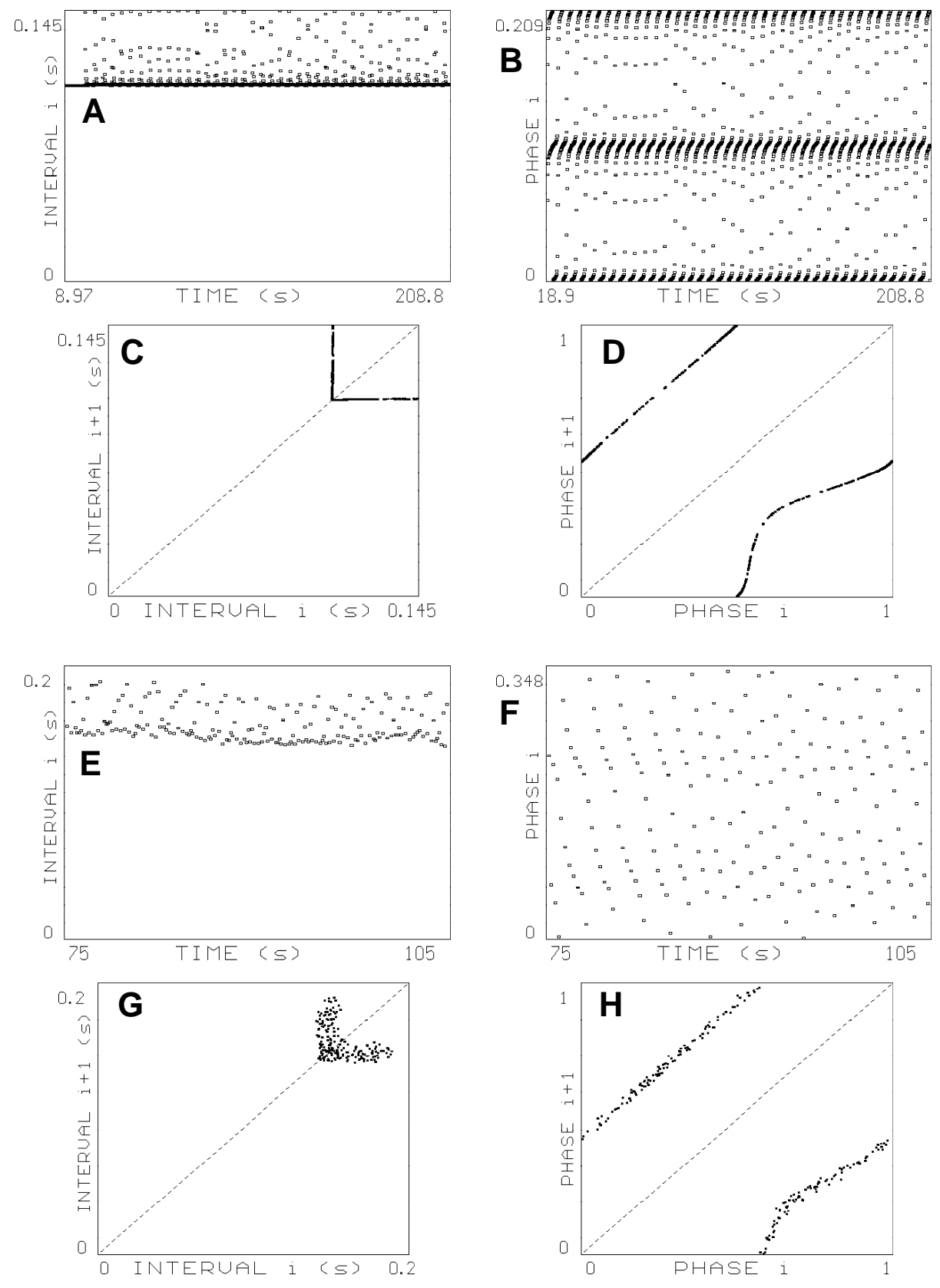

Figure 3: Walkthrough in simulation and SAO. Intervals (simulation: A, C; SAO: E, G) and phases (simulation: $\mathrm{B}, \mathrm{D}$; SAO: $\mathrm{F}, \mathrm{H})$. Intime $\left(T_{i}\right.$ vs. $\left.t_{i}\right)$ plots $(\mathrm{A}, \mathrm{E})$ show preferred minimum interval. Phases $(\mathrm{B}, \mathrm{F})$ alternate between long and short categories, which walk through the range of values. Interval return maps $(C, G)$ are ' $L$ ' shaped with TMelbows" on the diagonal. Phase return maps $(\mathrm{D}, \mathrm{H})$ shows continuous curves. Simulation parameters: $N / I=0.5, I=0.209 \mathrm{~s}$, $T=0.107 \mathrm{~s}, \bar{P}_{\text {syn }}=6.0 \times 10^{-9} \mathrm{~cm} / \mathrm{s}$. 


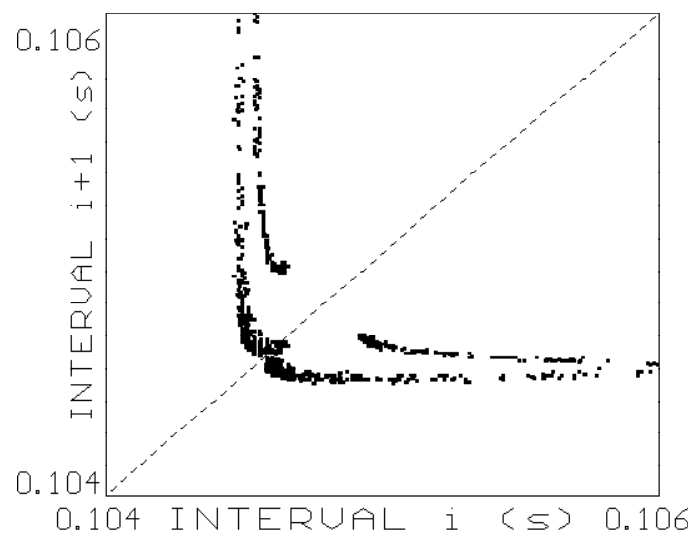

Figure 4: Expanded view of TMelbow" portion of interval return map in Figure ??(C) indicates Ænstructure.

interval return maps $(C, G)$ are ' $L$ ' shaped, with the elbow on the diagonal. Additionally, the phase return maps $(\mathrm{D}, \mathrm{H})$ contain points which fall along similar one-dimensional, invertible curves.

A magniÆediew of the elbow in (C) is presented in Figure ??. We see that the interval return map in Figures ??(C) and ?? is not invertible, and that there is a Ænstructure associated with it. Nearby points fall on topologically distant parts of whatever object is described by the map. This behavior is tentatively assigned to a pathway to chaos called collapse of the quasiperiodicity.

In the SAO, two different types of unpredictable (or TMmessy") behaviors were identiÆed: erratic and stammering [?, ?]. Erratic behavior occurs at relatively low presynaptic rates below 1:1 locking, and stammering occurs at high rates. We shall only comment on stammering here.

In the SAO, after the arrival of an inhibitory spike, there is a period of time when the neuron is unlikely to ÆreFor low input frequency, it will recover and be able to Ærbefore the next input. As the input frequency is increased, this ${ }^{\mathrm{TM}}$ recovered interval" is shortened, until it is a narrow ${ }^{\mathrm{TM}}$ Window" just around the input arrival time $(\phi \approx 0)$. Higher frequencies than that may cause the neuron to be silenced completely. 

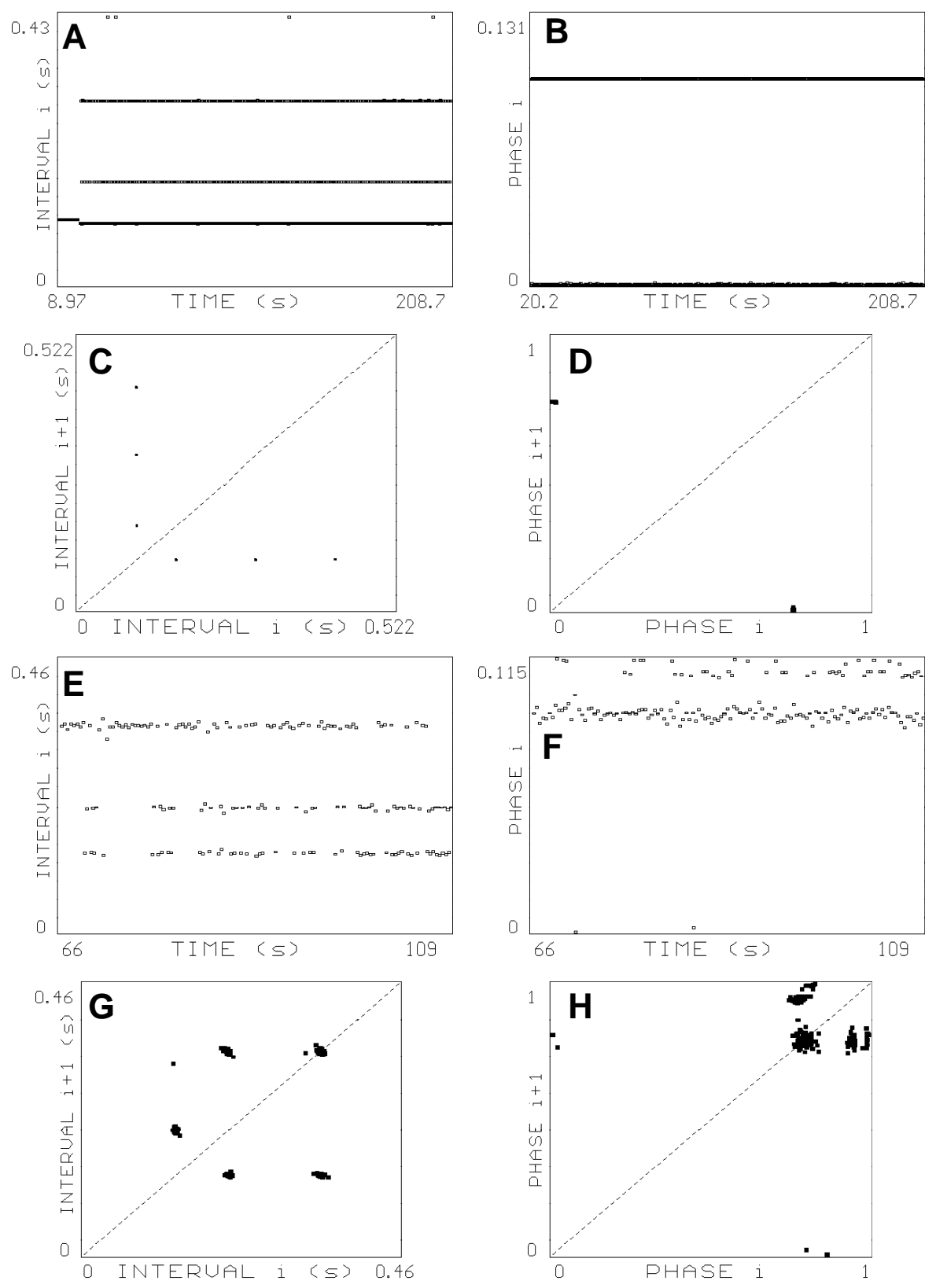

Figure 5: Stammering in simulation and SAO. Intervals (simulation: A, C; SAO: E, G) and phases (simulation: B, D; SAO: F, H). Intime plot (A) shows acceleratory effect of inhibition and extreme intervals which are multiples of driver. There are two classes of phases (B, F). Phases in the simulation alternate (D). Simulation parameters: $N / I=0.8, I=0.130 \mathrm{~s}, T=0.168 \mathrm{~s}$, $\bar{P}_{s y n}=7.0 \times 10^{-7} \mathrm{~cm} / \mathrm{s}$. 


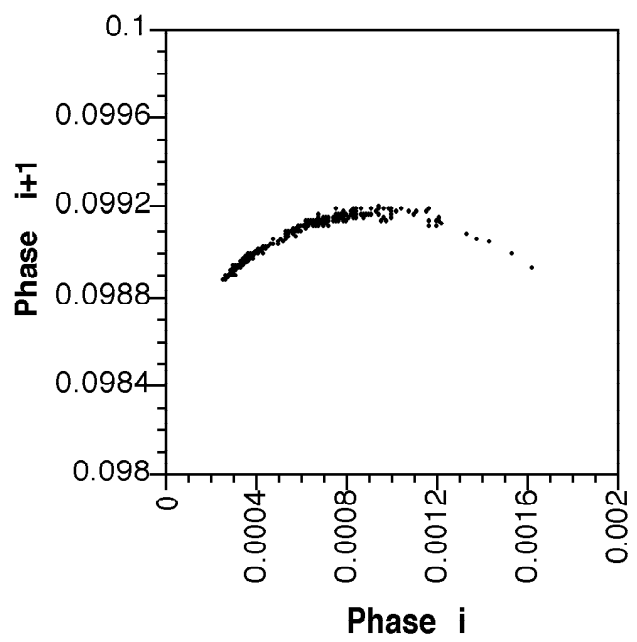

Figure 6: Enlargement of upper left ${ }^{\mathrm{TM}}$ point" from Figure ??(D) show the cluster has Æntructure, an extremum, and is not invertible.

This type of discretization occurs in both the SAO and the model, and is called windowing. The Øuctuationf excitability may be more complex, having multiple windows after an IPSP, separated by silent periods. Figure ?? corresponds to multiple-window situations (in both cases, with two windows per inhibitory spike). There are two windows visible in the phasetime plots $(\mathrm{B}, \mathrm{F})$, one around the time of arrival of the input (note $\phi=1 \equiv \phi=0$ ), and the other longer phase. The behavior is not regular, as evidenced by the interval return maps $(\mathrm{C}, \mathrm{G})$. Multiple windows were clear and relatively frequent in simulation data; the latter Ændingled to recognizing them also in SAO data where they were less apparent and had been missed initially.

Simulation results permit a more discerning dissection of the data, as can be seen in the enlargement of what appear to be points in the return maps (Figure ??). These TMpoints" have a structure, the result of an underlying deterministic process. These two islands express the same folding and stretching behavior that has been seen in sinusoidally forced autonomously oscillating squid giant axons [?]. Though it had been previously concluded that stammering in the SAO is the result of noise [?, ?], this suggests that there may be an underlying deterministic process which is ${ }^{\mathrm{TM}}$ washed out" by the noise. 


\section{Conclusions}

The permeability model examined here reproduced extensively the variety of behaviors exhibited by the preparation. Only the briefest of comparisons has been included here. This reproduction is important because simpler models, based on phase transition curves (PTCs) or leaky integrators, generate exclusively locked discharges, even when considerably modiÆe[?]. The model was also useful for improving our understanding of the messy behaviors. In erratic forms, it showed how common sliding is, both of intervals and phases (walkthroughs). In stammering, it demonstrated that tight clusters could have special structures suggesting chaotic behaviors within relatively small volumes. Both Ændingsoticed Ærsin the simulations, were subsequently identiÆein the SAO data (where they had been missed initially). The presence of chaotic dynamics in a deterministic model is strong evidence for it underlying the corresponding behaviors of the living preparation, reinforcing evidence from other tests [?].

This model is expressed in terms of physiologically relevant entities (e.g., permeabilities, pumps), and therefore allows the exploration of how each basic mechanism contributes to the genesis of each discharge form. Not only can such a model be analyzed more thoroughly, but it can also serve to guide biological experiments in parallel with theoretical investigations. Determining the computational implications of these dynamics can similarly proceed in parallel with experimental investigation of their functional consequences.

While typical ANN models emphasize smooth, continuous transfer functions across synapses, living neurons do not behave accordingly, exhibiting complex input/output relationships, which depend not only on average input frequency, but also on the timing pattern of input spike trains (such as regular, periodic, or aperiodic). This has been amply demonstrated for regularly spaced periodic input [?]. The continuing emphasis of ANN work on simple models entails the risk of the Æelldecoming irrelevant to the underlying neuroscience. 
We assert that the dynamics of individual processing elements, and the concommittent complexity of potential behaviors, is essential for the construction of ANNs whose performance is meant to approximate that of biological systems - that TM... knowledge of connectivity and synaptic weights alone are not sufÆcientb account for the operation and capabilities of neural networks..." [?]. The dynamics of individual neurons, and the temporal relationships among groups of them, are essential ingredients.

\section{References}

[1] J. P. Segundo, E. Altshuler, M. Stiber, and A. GarÆEnkeT,MPeriodic inhibition of living pacemaker neurons: I. Locked, intermittent, messy, and hopping behaviors," Int. J. Bifurcation

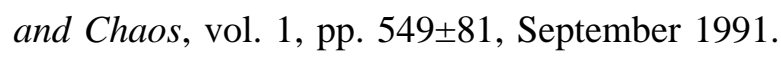

[2] M. Stiber, Dynamics of Synaptic Integration. PhD thesis, University of California, Los Angeles, 1992.

[3] G. Sugihara, D. Grace, J. Segundo, and M. Stiber in preparation, 1992.

[4] J. Segundo and D. Perkel, TMThe nerve cell as an analyzer of spike trains," in The Interneuron:

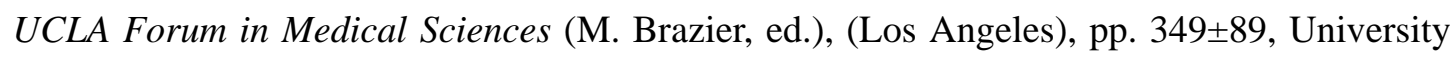
of California Press, 1969.

[5] Å. Edman, S. Gestrelius, and W. Grampp, TMAnalysis of gated membrane currents and mechanisms of Æringontrol in the rapidly adapting lobster stretch receptor neurone," J. Physiol.,

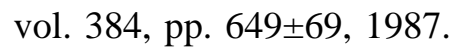

[6] G. Ermentrout and J. Rinzel, TMBeyond a pacemaker's entrainment limit: phase walk-through," Am. J. Physiol., vol. 246, pp. R102 \pm R106, 1984. (Regulatory Integrative Comp. Physiol. 15). 
[7] K. Aihara and G. Matsumoto, TMChaotic oscillations and bifurcations in squid giant axons," in

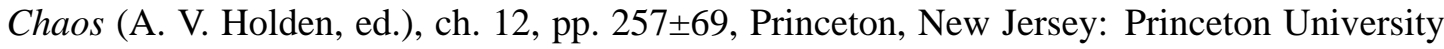
Press, 1986.

[8] N. Azmy, G. Yahiaoui, and J.-F. Vibert, ${ }^{\mathrm{TM}}$ Critical dependence of neural networks processing on between neuron delays," in Proceedings ICANN, (Helsinki), 1991. 